\title{
Collaborative care model improves self-care ability, quality of life and cardiac function of patients with chronic heart failure
}

\author{
C.Y. Hua ${ }^{1 *}$, Y. Huang ${ }^{2 *}$, Y.H. Su${ }^{3}$, J.Y. Bu ${ }^{3}$ and H.M. Tao ${ }^{4}$ \\ ${ }^{1}$ Department of Hematology and Rheumatology, The Fifth Affiliated Hospital of Sun Yat-Sen University, Zhuhai, Guangdong, China \\ ${ }^{2}$ Department of Cardiothoracic Surgery, The Fifth Affiliated Hospital of Sun Yat-Sen University, Zhuhai, Guangdong, China \\ ${ }^{3}$ Department of General Surgery I, The Fifth Affiliated Hospital of Sun Yat-Sen University, Zhuhai, Guangdong, China \\ ${ }^{4}$ Department of Nursing, The Fifth Affiliated Hospital of Sun Yat-Sen University, Zhuhai, Guangdong, China
}

\begin{abstract}
Chronic heart failure (CHF) is a common chronic disease that requires much care. This study aimed to explore the effects of collaborative care model (CCM) on patients with CHF. A total of 114 CHF patients were enrolled in this study, and were randomly and equally divided into two groups: control and experimental. Patients in the two groups received either usual care or CCM for 3 continuous months. The impacts of CCM on the self-care ability and quality of life were assessed using self-care of heart failure index and short form health survey 12, respectively. Further, cardiac function was assessed by measuring left ventricular ejection fraction (LVEF) and the level of N-terminal pro-B-type natriuretic peptide (NT-proBNP), and by the 6-min walking test. Clinical and demographic characteristics of patients in the control and CCM groups were statistically equivalent. Compared with usual care, CCM significantly enhanced self-care abilities of patients with CHF, including self-care maintenance, self-care management and self-care confidence (all $P<0.05$ ). The physical and mental quality of life was also significantly improved by CCM $(P<0.01$ or $P<0.05)$. Compared with usual care, CCM significantly increased the $L V E F(P<0.01)$, decreased the NT-proBNP level $(P<0.01)$, and enhanced exercise capacity $(P<0.001)$. In conclusion, CCM improved the self-care, quality of life and cardiac function of patients with CHF compared with usual care.
\end{abstract}

Key words: Collaborative care model; Chronic heart failure; Self-care; Quality of life; Cardiac function

\section{Introduction}

Heart failure is a final stage of heart disease, and it occurs when the heart muscle is unable to pump sufficient blood and oxygen to meet the needs of the body (1). Chronic heart failure ( $\mathrm{CHF}$ ) is a long-term condition of heart failure, which currently affects more than 23 million persons worldwide (2). CHF greatly decreases quality of life, reduces physical function, attention, executive function and memory (3). The symptoms of CHF commonly include weakness, fatigue, oliguria, memory loss, anxiety, headaches, insomnia, exertional dyspnea, orthopnea and edema (4). In many cases, behavioral modification, drug therapy and device treatment can significantly improve the outcomes (5). However, hospitalizations and readmissions for CHF remain major public health problems (6). The main goals of CHF treatment are the prolongation of life, and improvement of the quality of life.
The concept of collaborative care model (CCM) was developed to attempt to improve the quality of care (7). Over time, three core concepts, population-based care, measurement-based care and stepped care, are emphasized in CCM (8-11). In CCM, provision of care and health outcomes are defined based on a population of patients; each patient's progress is closely tracked using validated clinical rating scales (e.g., self-care index for heart failure); treatment is systematically adjusted, i.e., if patients do not improve as expected, initial adjustments can be made by the primary nurses $(7,12)$. To date, multiple studies have tested the effects of CCM on different diseases, such as depression and schizophrenia, and CCM has been consistently regarded as an effective care mode $(13,14)$. However, few reports have been focused on the interventional effects of CCM on CHF.

Correspondence: H.M. Tao: <taohongmei8113@126.com>

${ }^{*}$ These authors contributed equally to this study.

Received January 26, 2017 | Accepted June 22, 2017 
This study aimed to explore the effects of CCM on improving the care of patients with CHF.

\section{Material and Methods}

\section{Study design and participants}

A total of $114 \mathrm{CHF}$ patients were enrolled in this study, from November 2013 to January 2016. All participants were randomly and equally divided into a control group and an experimental group ( $n=57$ in each group). Patients had been diagnosed with CHF (systolic or diastolic dysfunction) with chest X-ray showing pulmonary edema, and New York Heart Association (NYHA) functional Class I-III. The left ventricular ejection fraction (LVEF) of patients in the two groups was lower than the normal range. Inclusion criteria for study participants were being older than 18 years old, and without dementia and acute coronary event in the prior 3 months. The spouse or the immediate family of these patients served as caregivers, and they accompanied the patients at the time of enrollment. This study was approved by the Sun Yat-Sen University Ethics Committee, and written informed consent was obtained from each adult before data collection.

Patients enrolled in this study were treated with spironolactone combined with metoprolol succinate. Spironolactone, a potassium-sparing diuretic, was used at a dose of $20 \mathrm{mg}$, three times a day. Metoprolol succinate (12.5$25 \mathrm{mg}$ ) was first used once a day, and then the dose was doubled every 2 weeks. If adverse effects were seen, including hypotension, fatigue, heart rate less than $55 \mathrm{bpm}$, fluid retention, II and III degree atrioventricular block, and myocardial infarction, dose doubling was delayed until the adverse effects disappeared.

\section{Intervention by usual care and CCM}

Patients in the control group were treated with usual care, including management of their symptoms, diet, exercise, medication and psychology.

CCM was administrated to the experimental group, and was monitored by the caregivers and primary nurses. First, a primary nurse evaluated the self-care ability and psychological status of patients. The primary nurse worked collaboratively with patients and their caregivers to establish individualized clinical and self-care goals. Second, the primary nurse handed out a health education booklet and video-tape, and introduced the type, dosage, curative effects and adverse reactions of common drugs to patients and their caregivers. The primary nurse supervised patients and their caregivers to manage symptoms and lifestyles, including weight, blood pressure and heart rate measurement, water and salt intake, reasonable exercise and sufficient sleeping. Finally, caregivers thereafter carried out follow-up telephone calls at each week after hospital discharge to provide feedback from patients regarding their adherence to overall CCM (1).

\section{Self-care of heart failure index (SCHFI)}

The SCHFI version 6.2 was carried out to measure selfcare of CHF patients as described previously (1). SCHFI is widely used for measuring the self-care behaviors of $\mathrm{CHF}$ patient. It consists of three subscales, the self-care maintenance scale, the self-care management scale and the self-care confidence scale. The self-care maintenance scale has 10 items measuring self-monitoring and treatment adherence, such as checking weight and ankle swelling, taking medication regularly, low sodium intake and appropriate exercise. The self-care management scale has 6 items measuring the abilities of symptom recognition, treatment implementation and treatment evaluation, such as, when dyspnea or ankle swelling occur and how to implement remedies. The self-care confidence scale has 6 items measuring the confidence of patients, such as the confidence to manage CHF. All items used a 4-point self-report scale and each of these three self-care subscales was standardized from 0 to 100 . Higher scores reflected better self-care.

\section{Short form health survey 12 (SF-12)}

The SF-12 was used as previously described to measure the quality of life of CHF patients (15). It consists of 12 items and two dimensions, physical component summary and mental component summary. The score of physical component summary items measures physical quality of life, which is influenced by physical health, physical functioning, role limitations and bodily pain (16). The score of mental component summary items measures mental quality of life, which is influenced by mental health, vitality, role limitations and social functioning. Both dimensions were standardized from 0 to 100 , and higher scores reflected better quality of life.

\section{LVEF measurement}

LVEF of CHF patients was measured by standard echocardiographic examinations using a Sonos 5500 ultrasound machine (Philips, The Netherlands) with a $2.5-\mathrm{Hz}$ transducer (17). The normal value of LVEF was $>50 \%$.

\section{N-terminal pro-B-type natriuretic peptide (NT-proBNP) measurement \\ NT-proBNP level was determined by a one-step sand-} wich enzyme-linked immunosorbent assay (ELISA) using the Novegent NT-proBNP ELISA kit (Chongqing Novegent Biotech Co., China), according to the manual (18). Before patients were transfused with packed red blood cells, $5 \mathrm{~mL}$ blood samples were collected into clot-tubes and used for measuring the NT-proBNP. Sera were extracted by centrifugation at $2500 \mathrm{~g}$ for $10 \mathrm{~min}$ at $4^{\circ} \mathrm{C}$, and then stored at $-80^{\circ} \mathrm{C}$ until analysis.

\section{6-min walking test (6MWT)}

The 6MWT is a simple method used for testing the exercise capacity of CHF patients. In brief, a marked 
hallway (30.5 m length) in the hospital was used to conduct the 6MWT. The patients were asked to walk back and forth along this hallway as far as possible for a period of $6 \mathrm{~min}$, at their own best pace but not to run (19). Each patient had a personal instructor to provide encouragement and announce the remaining time. The total distance walked within 6 min was recorded, and normal exercise capacity was designated by a walking distance between 375 to $449.5 \mathrm{~m}$.

\section{Statistical analysis}

Data are reported as means \pm SD or as $n(\%)$, as appropriate. Statistical analysis was performed using SPSS software (version 17.0 for Windows; SPSS Inc., USA). The differences between the control and experimental groups were analyzed by independent $t$-test or chi-square test. The obtained estimations were considered statistically significant if $\mathrm{P}<0.05$.

\section{Results}

\section{Clinical and demographic characteristics}

The mean patient age was $63.61 \pm 6.62$ and $65.44 \pm$ 5.79 years in the control and CCM groups, respectively. Patients in the control and CCM groups had similar educational levels, and more than $75 \%$ of patients had less than high school education. The values of LVEF in control and CCM patients were 41.82 \pm 16.01 and $42.06 \pm$ $13.81 \%$, respectively. In addition, patients were distributed throughout the four categories of NYHA functional class, although there was no class IV (symptomatic at rest) represented. Overall, there was no significant difference between the control group and CCM group in the mean age, gender, education, LVEF, and NYHA class, showing that the clinical and demographic characteristics of patients in the two groups were statistically equivalent (Table 1).

\section{CCM enhanced the self-care ability of CHF patients}

After 3 months of care, the SCHFI was performed to measure the self-care of CHF patients. The scores of selfcare maintenance, management and confidence in CCM group were all significantly higher than the control group (all $\mathrm{P}<0.05$; Figure 1). These results revealed that $\mathrm{CCM}$ enhanced the self-care ability of CHF patients.

\section{CCM enhanced the quality of life of CHF patients}

CCM significantly improved both the physical and mental quality of life, compared with the control group $(P<0.01$ or $P<0.05$; Figure 2$)$. Thus, we inferred that $\mathrm{CCM}$ also could improve the quality of life of CHF patients.

\section{CCM improved the cardiac function of CHF patients}

As shown in Figure 3, both the usual care and CCM significantly increased the LVEF $(P<0.05$ or $P<0.01)$, decreased the NT-proBNP level $(\mathrm{P}<0.05$ or $\mathrm{P}<0.01)$, and enhanced the exercise capacity $(P<0.05$ or $P<0.01)$, compared to baseline. More importantly, compared with usual care, CCM significantly increased the LVEF $(P<0.01)$, decreased the NT-proBNP level $(P<0.01)$, and enhanced

Table 1. Clinical and demographic characteristics of the control group (normal care, $n=57$ ) and the collaborative care model (CCM) group $(n=57)$ of chronic heart failure patients.

\begin{tabular}{lccc}
\hline & Control patients & CCM patients & P \\
\hline Age (years) & $63.61 \pm 6.62$ & $65.44 \pm 5.79$ & 0.119 \\
Gender & $36(63.16 \%)$ & $30(52.63 \%)$ & 0.343 \\
$\quad$ Male & $21(36.84 \%)$ & $27(47.37 \%)$ & \\
$\quad$ Female & $18(31.58 \%)$ & & \\
Education & $15(26.32 \%)$ & $17(29.82 \%)$ & 0.809 \\
$\quad$ Elementary & $12(21.05 \%)$ & $16(28.07 \%)$ & \\
Middle school & $8(14.04 \%)$ & $16(28.07 \%)$ & \\
Professional school & $4(7.02 \%)$ & $6(10.54 \%)$ & \\
High school & $41.82 \pm 16.01$ & $2(3.51 \%)$ & 0.932 \\
University degree & $11(19.30 \%)$ & $12.06 \pm 13.81$ & 0.747 \\
LVEF (\%) & $19(33.33 \%)$ & $22(38.60 \%)$ & \\
NYHA Class & $27(47.37 \%)$ & $23(40.35 \%)$ & \\
I & 0 & 0 & \\
II & & & \\
III & & & \\
IV & & & \\
\hline
\end{tabular}

Data are reported as means $\pm S D$ or number and percentages. CHF: chronic heart failure; LVEF: left ventricular ejection fraction measurement; NYHA: New York Heart Association. Statistical analysis was done with the $t$-test or chi-square test. 

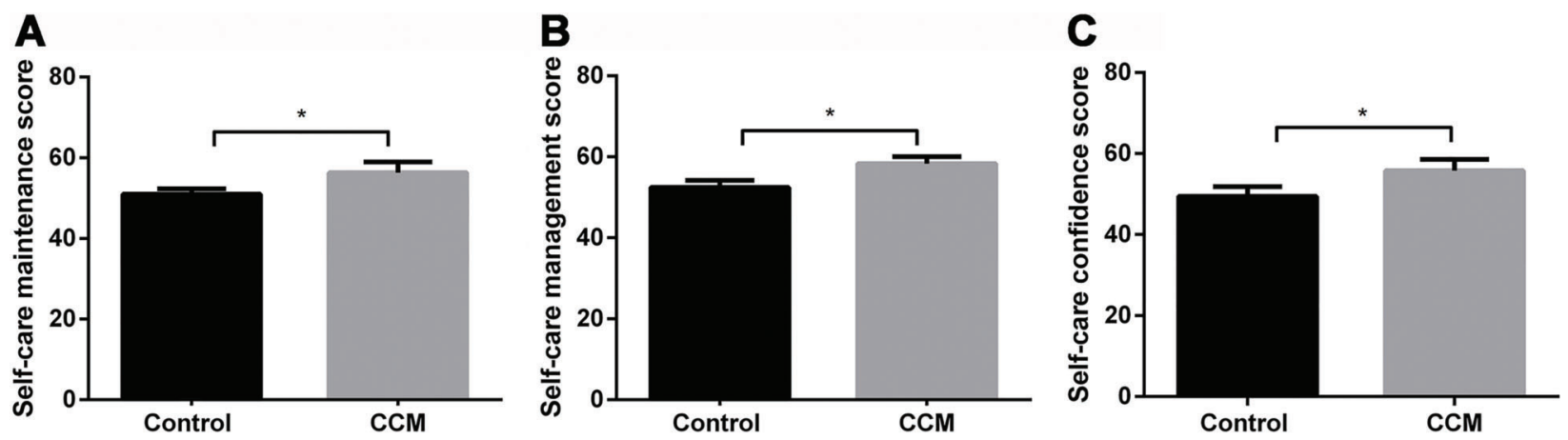

Figure 1. Comparison of $A$, self-care maintenance; $B$, self-care management, and $C$, self-care confidence between patients with chronic heart failure treated with usual care (Control) and with the collaborative care model (CCM). Data are reported as means $\pm S D$. ${ }^{*} P<0.05$ ( $t$-test).
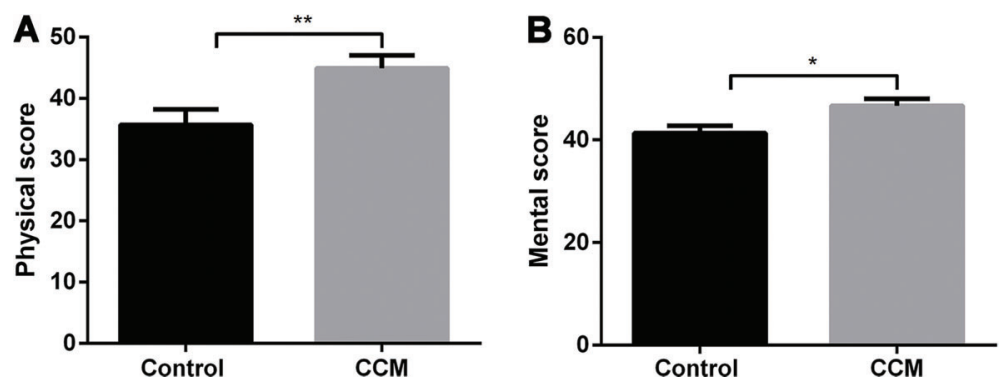

Figure 2. Comparison of $A$, physical quality of life, and $B$, mental quality of life between patients with chronic heart failure treated with usual care (Control) and with the collaborative care model (CCM). Data are reported as means $\pm \mathrm{SD}$. ${ }^{*} \mathrm{P}<0.05$; ${ }^{* *} \mathrm{P}<0.01$ (t-test).
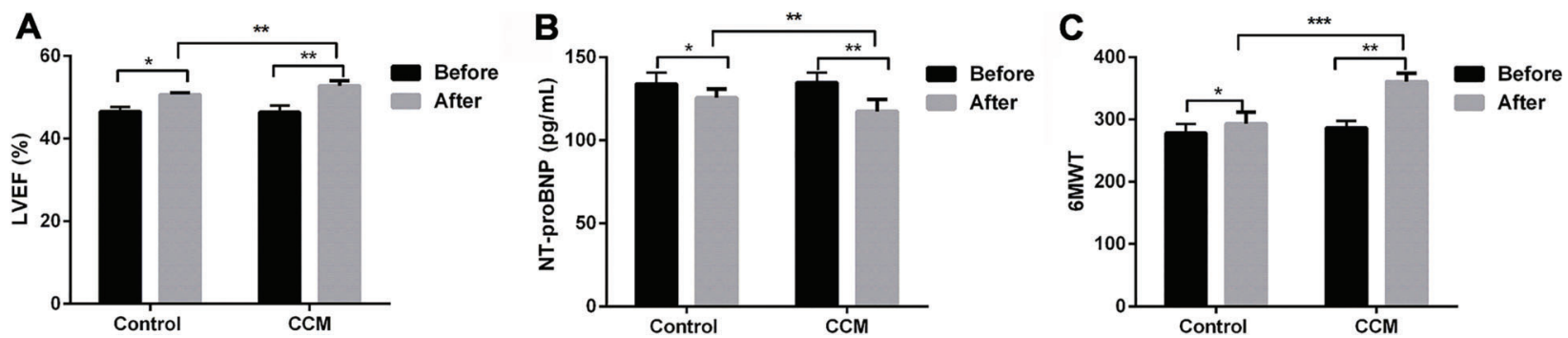

Figure 3. Comparison of $A$, left ventricular ejection fraction (LVEF); $B$, N-terminal pro-B-type natriuretic peptide (NT-proBNP) level, and $C$, exercise capacity between patients with chronic heart failure treated with usual care (Control) and with the collaborative care model (CCM). 6MWT: 6-min walking test. Data are reported as means $\pm \mathrm{SD}$. ${ }^{*} \mathrm{P}<0.05$; ${ }^{* *} \mathrm{P}<0.01$; ${ }^{* * *} \mathrm{P}<0.001$ ( $t$-test).

the exercise capacity $(\mathrm{P}<0.001)$. Therefore, $\mathrm{CCM}$ improved the cardiac function of CHF patients.

\section{Discussion}

$\mathrm{CHF}$ is a common chronic disease that requires much care. In the present study, we found that, compared with the control group, CCM significantly enhanced the selfcare ability, i.e., self-care maintenance, self-care management and self-care confidence. More surprisingly, physical and mental quality of life was significantly enhanced, and cardiac function was greatly improved by CCM.

CCM is regarded as an effective and efficient integrated care mode in many chronic diseases (7). In human immunodeficiency virus (HIV) and other specialty physical health care settings, HIV Translating Initiatives for Depression into Effective Solutions (HITIDES) intervention serves as one type of CCM, and it improves depression and HIV symptom outcomes (20). In attention-deficit hyperactivity disorder (ADHD), CCM appeared feasible and acceptable for the treatment of Hispanic children with ADHD and showed preliminary evidence of effectiveness (21). In terms of CHF, CCM also showed to be effective, and was associated with increased use of drug therapies and cardiovascular diagnostic tests and better outcomes compared with primary care alone (22). Asch et al. (23) demonstrated that $\mathrm{CCM}$ improved a wide range of processes of care for $\mathrm{CHF}$, including medical therapeutics, 
and education and counseling. Consistent with these previous studies, our findings showed that CCM could significantly improve self-care, quality of life and cardiac function of patients with CHF.

Self-care is defined as an individual's capacity to perform health promotion, disease prevention or maintenance, and even a capacity to address specific behaviors by changing the sense of well-being (24). It is essential to improve outcomes of patients with $\operatorname{CHF}(25,26)$. Several studies demonstrated that, collaboration with visiting nurses or a combination of telemonitoring counseling effectively promoted self-care, and then reduced readmission rates and promoted health (27-29). Katon et al. (30) reported that the self-care ability of patients with depression was enhanced by CCM with education encompassing self-monitoring, behavioral activation, goal setting and problem solving. Consistently, we found that CCM was essential for improving self-care abilities and outcomes in patients with CHF.

Quality of life and cardiac function, together with selfcare, are three important issues in CHF (16). Poor quality of life and cardiac function always accompany all stages of CHF. In addition, quality of life serves as a pivotal outcome measurement in any evaluation of treatment efficacy (31). Yehle et al. (32) found that with telemonitoring and home healthcare nurse visiting quality of life was remarkably improved. Moreover, exercising regularly has been found to be associated with major improvements in the quality of life and cardiac function $(33,34)$. Although the precise molecular mechanism(s) of action remain

\section{References}

1. Liou HL, Chen HI, Hsu SC, Lee SC, Chang CJ, Wu MJ. The effects of a self-care program on patients with heart failure. J Chinese Med Assoc 2015; 78: 648-656, doi: 10.1016/ j.jcma.2015.06.004.

2. Celik E, Cay S, Sensoy B, Murat S, Oksuz F, Cankurt T, et al. Heart Failure functional class associated with depression severity but not anxiety severity. Acta Cardiol Sinica 2016; 32: 55-61.

3. Dolansky MA, Schaefer JT, Hawkins MA, Gunstad J, Basuray A, Redle JD, et al. The association between cognitive function and objective adherence to dietary sodium guidelines in patients with heart failure. Patient Prefer Adherence 2016; 10: 233-241, doi: 10.2147/ppa.s95528.

4. Clark AM, Freydberg N, Heath SL, Savard L, McDonald M, Strain $L$. The potential of nursing to reduce the burden of heart failure in rural Canada: what strategies should nurses prioritize? Can J Cardiovasc Nurs 2008; 18: 40-46.

5. Man WD, Chowdhury F, Taylor RS, Evans RA, Doherty P, Singh SJ, et al. Building consensus for provision of breathlessness rehabilitation for patients with chronic obstructive pulmonary disease and chronic heart failure. Chron Respir Dis 2016; 13: 229-239, doi: 10.1177/1479972316642363.

6. Smit J, Adelborg K, Thomsen RW, Sogaard M, Schonheyder HC. Chronic heart failure and mortality in patients with unclear, physical exercise has been advocated as a nonpharmacological adjuvant to strengthen the conventional management and prevention of cardiovascular events (35). A series of clinical and experimental studies has demonstrated that an appropriate volume and intensity of exercise can ameliorate myocardial dysfunction through the improvement of maximum oxygen consumption, LVEF, LV diastolic and systolic volumes, ventilatory threshold, cardiac output and diastolic function (E/A ratio) (35-38). In the present study, the patients in the CCM group received telemonitoring from primary nurses and exercise training, which might partly explain the ameliorative effects of CCM on quality of life and cardiac function. Besides, the influence of caregivers' emotion on behavior of patients might contribute to these ameliorative effects. In one study of 27 patients with CHF and their family caregivers, a positive orientation toward solving problems in caregivers was a significant predictor of improved quality of life in patients (39).

As a limitation, the patients enrolled in this study had mild CHF, as evidenced by preserved LVEF values, low NT-proBNP values, and no NYHA functional class IV represented.

In conclusion, the current data showed that CCM improved the self-care, quality of life and cardiac function of patients with mild CHF when compared with usual care. Whether CCM would also improve NYHA class IV patients still needs to be investigated. Further research is also needed to understand the underlying mechanism of this effect. community-acquired Staphylococcus aureus bacteremia: a population-based cohort study. BMC Infect Dis 2016; 16: 227, doi: 10.1186/s12879-016-1570-7.

7. Katon W. Collaborative depression care models: from development to dissemination. Am J Prevent Med 2012; 42: 550-552, doi: 10.1016/j.amepre.2012.01.017.

8. Wagner EH, Austin BT, Von Korff M. Organizing care for patients with chronic illness. Milbank Quarter 1996; 74: 511544, doi: 10.2307/3350391.

9. Katon WJ, Seelig M. Population-based care of depression: team care approache to improving outcomes. J Occup Environ Med 2008; 50: 459-467, doi: 10.1097/JOM.0b013e 318168 efb7.

10. Von Korff M, Tiemens B. Individualized stepped care of chronic illness. West J Med 2000; 172: 133-137, doi: 10.1136/ ewjm.172.2.133.

11. Trivedi $\mathrm{MH}$. Treating depression to full remission. $J$ Clin Psychiatry 2009; 70: e01.

12. Unützer J, Harbin $\mathrm{H}$, Schoenbaum M, Druss B. The collaborative care model: An approach for integrating physical and mental health care in Medicaid health homes. Health Home, Information Resource Center 2013; 1-13.

13. Simon G. Collaborative care for mood disorders. Curr Opin Psychiatry 2009; 22: 37-41, doi: 10.1097/YCO.0b013e328313e3fo. 
14. Neville C. Collaborative care approaches for people with severe mental illness. Clin Nurse Spec 2015; 29: 143-144, doi: 10.1097/NUR.0000000000000127.

15. Ware J Jr, Kosinski M, Keller SD. A 12-Item Short-Form Health Survey: construction of scales and preliminary tests of reliability and validity. Med Care 1996; 34: 220-233, doi: 10.1097/00005650-199603000-00003.

16. Vellone E, Chung ML, Cocchieri A, Rocco G, Alvaro R, Riegel B. Effects of self-care on quality of life in adults with heart failure and their spousal caregivers: testing dyadic dynamics using the actor-partner interdependence model. J Family Nurs 2014; 20: 120-141, doi: 10.1177/10748407 13510205.

17. Hu W, Jin X, Zhang P, Yu Q, Yin G, Lu Y, et al. Deceleration and acceleration capacities of heart rate associated with heart failure with high discriminating performance. Sci Rep 2016; 6: 23617, doi: 10.1038/srep23617.

18. Mehrzad V, Khajouei AS, Fahami E. Correlation of $\mathrm{N}$-terminal pro-B-type natriuretic peptide levels and cardiac magnetic resonance imaging $\mathrm{T}^{*}$ in patients with betathalassaemia major. Blood Transfus 2016; 14: 1-5, doi: 10.2450/2016.0120-15.

19. Chen CA, Chang $\mathrm{CH}$, Lin MT, Hua YC, Fang WQ, Wu MH, et al. Six-minute walking test: normal reference values for Taiwanese children and adolescents. Acta Cardiol Sinica 2015; 31: 193-201.

20. Pyne JM, Fortney JC, Curran GM, Tripathi S, Atkinson JH, Kilbourne AM, et al. Effectiveness of collaborative care for depression in human immunodeficiency virus clinics. Arch Intern Med 2011; 171: 23-31, doi: 10.1001/archinternmed. 2010.395.

21. Myers K, Stoep AV, Thompson K, Zhou C, Unutzer J. Collaborative care for the treatment of Hispanic children diagnosed with attention-deficit hyperactivity disorder. Gen Hosp Psychiatry 2010; 32: 612-614, doi: 10.1016/j.genhosp psych.2010.08.004.

22. Lee DS, Stukel TA, Austin PC, Alter DA, Schull MJ, You JJ, et al. Improved outcomes with early collaborative care of ambulatory heart failure patients discharged from the emergency department. Circulation 2010; 122: 1806-1814, doi: 10.1161/circulationaha.110.940262.

23. Asch SM, Baker DW, Keesey JW, Broder M, Schonlau M, Rosen M, et al. Does the collaborative model improve care for chronic heart failure? Med Care 2005; 43: 667-675, doi: 10.1097/01.mlr.0000167182.72251.a1.

24. Orem D. Nursing: concepts of practice. 6 th edn. St Louis: Mosby; 2001.

25. Riegel B, Dickson VV, Faulkner KM. The situation-specific theory of heart failure self-care: revised and updated. J Cardiovasc Nurs 2016; 31: 226-235, doi: 10.1097/jcn. 0000000000000244.

26. Barnason S, Zimmerman L, Young L. An integrative review of interventions promoting self-care of patients with heart failure. J Clin Nurs 2012; 21: 448-475, doi: 10.1111/j.13652702.2011.03907.x.

27. Duffy JR, Hoskins LM, Chen M-C. Nonpharmacological strategies for improving heart failure outcomes in the community: a systematic review. J Nurs Care Quality 2004; 19: 349-360, doi: 10.1097/00001786-200410000-00011.

28. Tsuyuki RT, McKelvie RS, Arnold JMO, Avezum A Jr, Barretto AC, Carvalho AC, et al. Acute precipitants of congestive heart failure exacerbations. Arch Intern Med 2001; 161: 2337-2342, doi: 10.1001/archinte.161.19.2337.

29. Inglis S. Structured telephone support or telemonitoring programmes for patients with chronic heart failure. J Evidence-Based Med 2010; 3: 228-228, doi: 10.1111/j.1756-5391. 2010.01104.x.

30. Katon WJ, Lin EH, Von Korff M, Ciechanowski P, Ludman EJ, Young B, et al. Collaborative care for patients with depression and chronic illnesses. New Engl J Med 2010; 363: 2611-2620, doi: 10.1056/NEJMoa1003955.

31. Dracup K, Walden J, Stevenson L, Brecht M. Quality of life in patients with advanced heart failure. J Heart Lung Transplant 1991; 11: 273-279, doi: 10.1016/S0147-9563(96)80061-6.

32. Yehle KS, Plake KS, Nguyen P, Smith D. Health-related quality of life in heart failure patients with varying levels of health literacy receiving telemedicine and standardized education. Home Healthcare Now 2016; 34: 267-272, doi: $10.1097 /$ nhh. 0000000000000384 .

33. Kavanagh T, Myers MG, Baigrie RS, Mertens DJ, Sawyer P, Shephard R. Quality of life and cardiorespiratory function in chronic heart failure: effects of 12 months' aerobic training. Heart 1996; 76:; 42-49, doi: 10.1136/hrt.76.1.42.

34. Hambrecht R, Niebauer J, Fiehn E, Kälberer B, Offner B, Hauer K, et al. Physical training in patients with stable chronic heart failure: effects on cardiorespiratory fitness and ultrastructural abnormalities of leg muscles. J Am Coll Cardiol 1995; 25: 1239-1249, doi: 10.1016/0735-1097(94) 00568-B.

35. Lew JK, Pearson JT, Schwenke DO, Katare R. Exercise mediated protection of diabetic heart through modulation of microRNA mediated molecular pathways. Cardiovasc Diabetol 2017; 16: 10, doi: 10.1186/s12933-016-0484-4.

36. Byrkjeland R, Njerve IU, Anderssen S, Arnesen H, Seljeflot I, Solheim S. Effects of exercise training on $\mathrm{HbA} 1 \mathrm{c}$ and VO2peak in patients with type 2 diabetes and coronary artery disease: A randomised clinical trial. Diabetes Vasc Dis Res 2015; 12: 325-333, doi: 10.1177/1479164115590552.

37. Hollekim-Strand SM, Bjorgaas MR, Albrektsen G, Tjonna $A E$, Wisloff $U$, Ingul $C B$. High-intensity interval exercise effectively improves cardiac function in patients with type 2 diabetes mellitus and diastolic dysfunction: a randomized controlled trial. J Am Coll Cardiol 2014; 64: 1758-1760, doi: 10.1016/j.jacc.2014.07.971.

38. De Angelis KL, Oliveira AR, Dall'Ago P, Peixoto LR, Gadonski G, Lacchini S, et al. Effects of exercise training on autonomic and myocardial dysfunction in streptozotocindiabetic rats. Braz J Med Biol Res 2000; 33: 635-641, doi: 10.1590/S0100-879X2000000600004.

39. Kurylo M, Elliott TR, DeVivo L, Dreer LE. Caregiver social problem solving abilities and family member adjustment following congestive heart failure. J Clin Psychol Med Settings 2004; 11: 151-157, doi: 10.1023/B:JOCS.00000 37609.23007.24. 